\title{
Lifelogging for Hidden Minds: Interacting Unconsciously
}

\author{
Huang-Ming Chang, Leonid Ivonin, Wei Chen, and Matthias Rauterberg \\ Industrial Design, Eindhoven University of Technology, Den Dolech 2, \\ 5612 AZ Eindhoven, The Netherlands
}

\begin{abstract}
In this paper, we propose a conceptual design of an entertainment system, which would utilize a combination of lifelogging and bio-signal measuring techniques for capturing unconscious communication flow coming from a user. We expect this system could interpret the user's unconsciousness and generate an emotionally rich feedback framed in a narrative, which would be tailored according to the universal symbolic patterns. This concept aims to explore a new area of entertainment computing. The discussed system looks promising to proceed with future work.
\end{abstract}

Keywords: Unconsciousness, Symbol, Bio-signal, Emotion, Lifelogging, Entertainment, Narrative, Interaction.

\section{Introduction}

According to [4], human to human interaction is always induced by both levels of cognition: conscious level (e.g., knowledge) and un- or subconscious level (e.g. emotions). As illustrated in Figure 1, traditionally for interaction with products, people manipulate products based on explicit mental models that is built by their consciousness [3]. Products, in turn, provide a feedback that is enclosed in logical messages intended for users' conscious understanding [4]. Affective Computing has addressed the importance of emotion in interaction between user and products [5]. To make the next step in enhancement of user experience, we intend to bring userproduct interaction to a high-dimensional level of human minds: unconsciousness. Therefore, we propose a concept of entertainment system that allows users to interact with it unconsciously.

To achieve this goal, it is proposed to utilize physiological data from users to detect implicit traces of unconscious behaviors. On the other hand, the system would provide a feedback, which could reach users' unconsciousness. According to Jung [1], universal symbolic content (e.g. Mandala) could be one of the communicative media that might get in touch with human's unconsciousness. The system aims at offering users experience, which enables both consciousness and unconsciousness to be unified and mutually enhancing. Moreover the system would present to users an opportunity of sharing emotional experience with other users, thus improving humanto-human communication. 


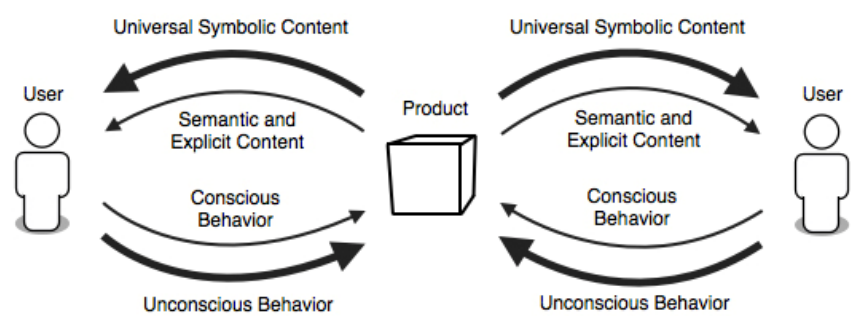

Fig. 1. Human-product interaction: traditional (thin line) and new approaches (bold line)

\section{The Concept of a System for Unconsciousness Interaction}

There are obviously two main challenges in the design of such a system. The first challenge is to access and interpret user's unconsciousness. We see an opportunity to overcome this challenge by recording physiological signals, which exhibit traces of human unconsciousness. The second challenge is to generate a feedback, which could reach users' unconsciousness. Universal symbolic content seems to be a good media type for this purpose, which would be able to reach human unconsciousness. Therefore, the correlation among psychological status, physiological status, and universal symbolic contents should be further clarified.

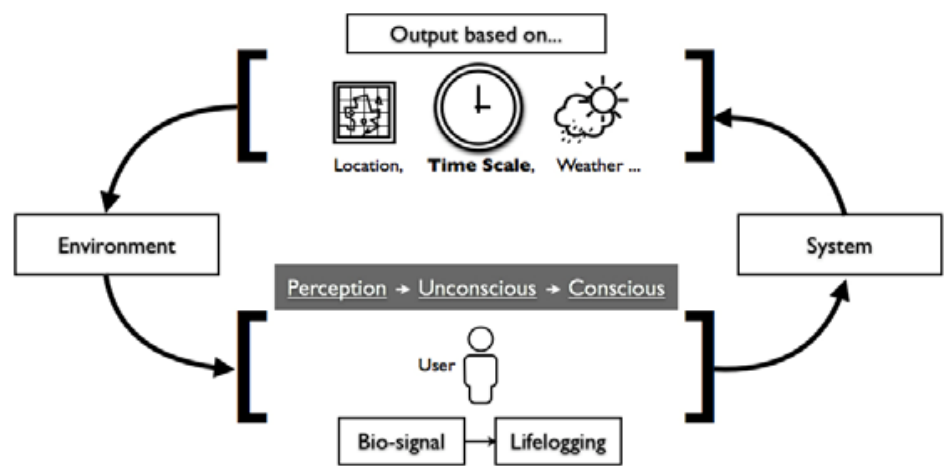

Fig. 2. The conceptual design of the entertainment system based on Kansei Mediation interaction model

As shown in Figure 2, this system would consist of a wearable lifelogging device, which would include sensors for both recording user's physiological signals and user's environment. The lifelogging device would selectively collect snapshots of the user's environment only at the moments of time that for some reason important for the user, even if the user consciously does not think that a moment is important. The selection of the moment to make a snapshot of reality (including photo or video, sound, location, etc.) would be automated based on the physiological signals of the user obtained from the sensors. The system then is able to analyze these materials and generate interpretation of them. Based on the mapping between physiological status 
and universal symbolic content, the system would generate a symbolic pattern. This feedback can be woven into the context of everyday life and be implemented in various applications. The reaction of the system would be merged into the background of the environment that will not grab users' full attention, but can be perceived unconsciously.

\subsection{Lifelogging and Bio-Signal Components}

We see a good opportunity for application of the lifelogging to enable people to exposure their unconsciousness triggered by first-person experience. Lifelogging applications use different kinds of sensors, which can be considered as an objective source of information about the environment, comparing to humans perception. Therefore the lifelogging will allow capturing the data from the environment as stimuli that influence a user's psychological status. However, the key issue is to choose a proper moment to trigger this process. Our answer to this is measuring physiological signals. Therefore, this device will also monitor a user's physiological status as a reference to observe implicit psychological status of the user.

According to Rauterberg [6], the human unconscious is represented by the genetic reproduction system, the peripheral nervous system (PNS) and the central nervous system (CNS). These three distinct systems contribute to unconscious information processing. Within each of them it is possible to find certain subsystems, which have direct relation to emotional feelings. Therefore it is proposed to use physiological aspects of human emotions for an interpretation of the human unconscious. This idea would be a starting point in the design of an entertainment system, which is able to understand human unconsciousness. Quantified physiological signals of human body would serve as an input data for the system and would provide an insight into the current state of mind of the user.

There is a number of physiological metrics, which are used to infer information regarding emotional state of a person, for example, galvanic skin resistance, respiration, temperature, and heart rate [2]. These metrics are promising for monitoring non-conscious activities because they cannot be easily controlled. Taking into account that the lifelogging component of the system, which requires information about the current psychological state of the user, is wearable, bio-sensors for measurement of physiological signals have to be non-intrusive and wearable as well. The data obtained from bio-sensors would allow automatically trigger lifelogging at the points of time, which have significant relevance for human's unconsciousness.

\subsection{Narratives and Other Media as Feedback}

As a potential application of an entertainment system, we intend to represent user's unconsciousness via generating a narrative that consists of lifelogging snapshots and universal symbolic contents. Since one might not be aware of when and where the snapshots were taken by the lifelogging device, this would consequently arouse their curiosity. "Curiosity is a state in which one's interest is heightened, leading to exploration" [7]. Especially when this concept is put into the context of everyday routine, which might be boring to some of us, the narratives could extract unconscious experience that would surprise us. So the concept of the system by itself might 
provoke curiosity, but it is important to enable the system to convey the implicit emotional feelings to the users. Depending on how the system organizes the story and on the ingredients, which the system adds to the narratives, the emotional reaction would vary. Previous research has exemplified how to lead users to the emotions of boredom and curiosity [7], which reveals the potential of using narratives for conveying emotions.

\section{Conclusion and Future Work}

We consider the proposed concept of an entertainment system as a promising direction for future research, which would lead to an unconscious mode of human and product interaction. However this ambitious goal poses a number of research questions: (1) Mapping of physiological signals and psychological states of users; (2) Mapping of psychological states of users and universal symbolic content; (3) Practical application of the system in entertainment.

Acknowledgement. This work was supported in part by the Erasmus Mundus Joint Doctorate in Interactive and Cognitive Environments, which is funded by the EACEA Agency of the European Commission under EMJD ICE FPA n 2010-0012.

\section{References}

1. Jung, C.G.: Man and His Symbols, vol. 432 (1968)

2. Khalili, Z., Moradi, M.: Emotion recognition system using brain and peripheral signals: using correlation dimension to improve the results of EEG (2009)

3. Nakatsu, R., Rauterberg, M.: Entertainment computing: Inaugural Editorial. Entertainment Computing 1, 1-7 (2009)

4. Nakatsu, R., Rauterberg, M., Salem, B.: Forms and theories of communication: from multimedia to Kansei mediation. Multimedia Systems 11, 304-312 (2006)

5. Picard, R.W.: Affective Computing. Mit Media Laboratory Perceptual Computing Section 321 (1995)

6. Rauterberg, M.: Emotions: The voice of the unconscious. In: Yang, H., Malaka, R., Hoshino, J., Han, J. (eds.) ICEC 2010. LNCS, vol. 6243, pp. 205-215. Springer, Heidelberg (2010)

7. van Aart, J., Bartneck, C., Hu, J., Rauterberg, M., Salem, B.: How to behave as Alice in Wonderland - about boredom and curiosity. Entertainment Computing 1, 125-137 (2010) 